\title{
Loop extrusion drives very different dynamics for Rouse chains in bulk solutions and at interfaces
}

\author{
Tetsuya Yamamoto ${ }^{1,2}$, Takahiro Sakaue ${ }^{2,3}$ and Helmut Schiessel ${ }^{4}$ \\ 1 Department of Materials Physics, Nagoya University - Furo-cho, Chikusa-ku, Nagoya 464-8603, Japan. \\ 2 PRESTO, Japan Science and Technology Agency (JST) - 4-1-8 Honcho, Kawaguchi, Saitama, 332-0012, Japan. \\ 3 Department of Physics and Mathematics, Aoyama Gakuin University - 5-10-1 Fuchinobe, Chuo-ku, Sagamihara, \\ Kanagawa 252-5158, Japan. \\ 4 Instituut-Lorentz for Theoretical Physics, Leiden University - Niels Bohrweg 2, Leiden, 2333 CA, The Netherlands.
}

\author{
PACS 87.15. He - Dynamics and conformational changes \\ PACS 87.16.Uv - Active transport processes \\ PACS $68.47 . \mathrm{Pe}$ - Langmuir-Blodgett films on solids; polymers on surfaces; biological molecules \\ on surfaces
}

\begin{abstract}
The loop extrusion theory predicts that the loops of chromosomes are produced by cohesin molecules that uni-directionally extrude a chromatin fiber. We here use an extension of the Rouse model to predict the chain conformational dynamics driven by the loop extrusion process. Our theory predicts that in a bulk solution, the mean square distance between the starting and ending sites of the loop extrusion process decreases with a constant rate. This is because the tension generated by the loop extrusion process drives the displacement of the starting site towards the ending site. In contrast, when the cohesin is entrapped at an interface, the mean square distance does not decrease until the tension generated by the loop extrusion process arrives at the ending site. This theory highlights the fact that the chain dynamics strongly depends on the mobility of the chain segments bound by cohesin.
\end{abstract}

Introduction. - In the interphase, chromosomes are composed of so-called topologically associated domains (TADs), contiguous regions of enriched contact frequency that are isolated from neighboring regions $[1,2]$. In many cases, there are peaks of contact frequency at the boundaries of TADs, implying that TADs are loops of chromatin fiber [3]. Recent theory predicts that the chromatin loops are produced by the loop extrusion process, with which cohesin, a ring-shaped protein complex, uni-directionally extrudes the chromatin fiber until it collides with a protein factor, called CTCF (CCCTC-binding factor) [4, 5]. CTCF proteins at the boundary of TADs are oriented towards the interior of the domains [3]. Indeed, most loops are lost when the loading of cohesin to chromosomes is suppressed [6]. The loop extrusion theory captures the features of TADs for a window of parameters $[4,5]$.

Typically, to start transcription (that is the process to synthesize messenger RNA), the binding of two distant DNA sequences, such as promoters and enhancers, are necessary. The binding rate of these sequences are governed by the chain conformational dynamics of the chromatin fiber between the sequences $[7,8]$. How does the loop extrusion process modulate the chain dynamics? Simulation models of chromatin fibers, on which cohesin drives a loop extrusion process, were constructed to predict the contact frequency map $[4,5]$. An analytical theory by using a simple model may be useful to understand the feature of the chain dynamics that is driven by the loop extrusion process.

Many of the simulations treat chromatin fibers in a uniform solution $[4,5]$. However, recent experiments have shown that droplets of the condensate of transcriptional activators and coactivators are stabilized by phase separation and superenhancers, DNA regions condensed with enhancer sequences, are associated with the droplets $[9,10]$. Other regions tend to be excluded from the droplets [11]. These experiments imply that chromatin stabilizes a structure analogous to microemulsions and cohesin may extrude chromatin at the surface of the droplets. Recent simulations of the loop extrusion process on chromosomes that show microphase separation did not emphasize the difference of the dynamics between chains in the bulk and 
a.

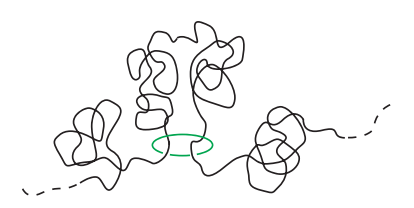

b.

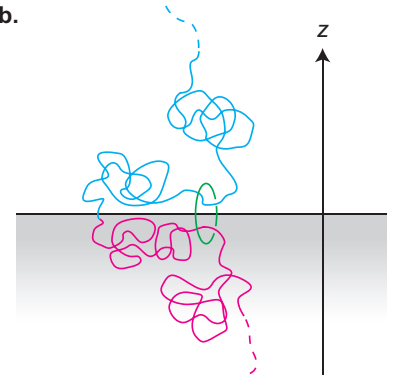

Fig. 1: Chains in a bulk solution (a) and at an interface (b). Cohesin (the green hoop) is loaded on the chain and drives the loop extrusion process. In the bulk solution, all the chain segments are freely mobile, whereas at the interface, the chain segment occupied by the cohesin is trapped at the interface because this cohesin binds the two segments that favor different environments. We set the $z$-direction to be the normal to the interface.

those at an interface [12]. It is thus of interest to theoretically predict the chain dynamics of chromatin in the bulk and at an interface.

We here use an extension of the Rouse model to predict the mean square of the distance between the starting and ending sites of the loop extrusion process, when a cohesin is loaded on the starting site at $t=0$. Our theory predicts that in the bulk solution, the mean square distance decreases with constant rate as soon as the cohesin starts the loop extrusion process. This reflects the fact that the loop extrusion process stretches the chain and generates the elastic force that displaces the starting site towards the ending site. In contrast, at an interface, the mean square distance does not change until the tension generated by the loop extrusion process arrives at the chain end. This happens because the starting site, which is embraced by cohesin, cannot escape from the interface and the mean square distance thus does not change until the ending site moves towards the starting site.

Model. - We treat the dynamics of a very long chain in a bulk solution and at an interface, see fig. 1. We use the bead-spring model [13] that treats the chain as beads that are linearly connected by springs, see fig. 2. The chain has a region, composed of $N$ beads, that is delineated by two CTCF molecules (of the converging orientations [3]). Cohesin is loaded on the chain from a site in the vicinity of a CTCF molecule, where a cohesin loader, Nipbl, is localized. The cohesin then starts extruding the chain with a constant rate $\tau_{c}^{-1}$ until it reaches the other CTCF site. The cohesin embraces two beads - one is the bead at the starting site and another changes as the cohesin extrudes the chain. At each extrusion process, cohesin pulls the chain so that the bead at the nearest neighbor displaces to the position of the cohesin, while the cohesin keeps embracing the starting site, see fig. 2 . The asymmetric extrusion has been observed by single molecule techniques for condensin $[14,15]$, whose struc-

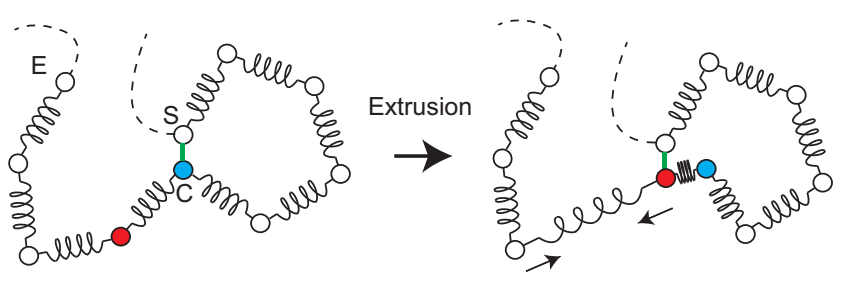

Fig. 2: We use the bead and spring model to treat the dynamics of the chain. A cohesin (shown by the green bar) is loaded on the chain from a site $(\mathrm{S})$ and embraces the starting site and the $m$-th site (C). At each extrusion process, the cohesin applies forces to capture the bead at the nearest neighbor. The cohesin operates the loop extrusion process with a constant rate $\tau_{\mathrm{c}}^{-1}$ until it reaches the ending site $(\mathrm{E})$.

ture is analogous to cohesin, and it is also implied from Hi-C experiments [16]. We predict the mean square of the distance between the starting and ending sites of the loop extrusion process when a cohesin is loaded on the chain at $t=0$, assuming that the chain is in the equilibrium for $t<0$.

The chain at the interface is composed of two types of blocks, one tends to be associated with the droplet and one is excluded from the droplet. Although the genomic position of the starting site may depend on experimental systems, cohesin may eventually arrive at the boundary between the two blocks. Because cohesin embraces the beads of different type, it is entrapped at the interface, analogous to surfactants. To highlight the role played by the interface in the dynamics of the chain, we treat the case in which the starting site is at the boundary between the two types of blocks. In the bulk solution, the system is isotropic and thus it is enough to treat the dynamics of the beads in one direction, see fig. 1a. At the interface, the bead that is bound by the cohesin does not move in the normal to the interface (the $z$-direction); the characteristics of the interface is manifested in the dynamics of the chain in the $z$-direction, see fig. $1 \mathbf{b}$. In the following, we thus treat the dynamics of the beads in the $z$-direction.

We use an extension of the Rouse model [13] to treat the dynamics of the chain. The Rouse model takes into account the connectivity of the chain, but neglects the hydrodynamic interactions and excluded volume interactions between chain segments. With this model, the position $z_{n}(t)$ of the $n$-th bead in the chain is derived by using the force balance equation

$$
\zeta \frac{\partial}{\partial t} z_{n}(t)=k \frac{\partial^{2}}{\partial n^{2}} z_{n}(t)+F_{m}(t) \delta_{n m}+f_{n}(t)
$$

where $n(=1,2, \cdots, N)$ counts the beads from the starting site to the ending site. The left side of eq. (1) is the friction force between the bead and the solvent. The first term of the right side is the elastic force generated by the springs that are connected to the bead. The second term is the force generated by the loop extrusion process when the cohesin is at the $m$-th bead $\left(\delta_{m n}=1\right.$ if $m=n$ and 
0 otherwise). The third term is the force caused by the random collisions of solvent molecules with the bead. $\zeta$ is the friction constant of the bead and $k\left(=3 k_{\mathrm{B}} T / b^{2}\right)$ is the spring constant of the springs that connect the beads $\left(k_{\mathrm{B}}\right.$ is the Boltzmann constant, $T$ is the absolute temperature, and $b$ is the Kuhn length). $f_{n}(t)$ is the Gaussian random force with $\left\langle f_{n}(t)\right\rangle=0$ and $\left\langle f_{m}(t) f_{n}\left(t^{\prime}\right)\right\rangle=2 \zeta k_{\mathrm{B}} T \delta_{m n} \delta(t-$ $\left.t^{\prime}\right)$. The form of the force $F_{m}(t)$ due to the loop extrusion process is shown later, see eqs. (6) and (7). Eq. (1) predicts that the relaxation time of a chain composed of $N$ segments is the Rouse time [13] defined by

$$
\tau_{N}=\frac{N^{2} \zeta}{\pi^{2} k}
$$

The Green function of eq. (1) is defined by the solution of the equation [17]

$$
\frac{\partial}{\partial t} G(n, m, t)=\frac{k}{\zeta} \frac{\partial^{2}}{\partial n^{2}} G(n, m, t)+\delta_{m n} \delta(t) .
$$

The solution of eq. (3) has the form

$$
G(n, m, t)=\left(\frac{\zeta}{4 \pi k t}\right)^{1 / 2} \mathrm{e}^{-\zeta(n-m)^{2} /(4 k t)}
$$

for an infinitely long chain. The solution of eq. (1) thus has the form

$z_{n}(t)=z_{n}(0)+\frac{1}{\zeta} \int_{0}^{t} d t^{\prime} G\left(n, m, t-t^{\prime}\right) F_{m}\left(t^{\prime}\right)+r_{n}(t)$,

where $r_{n}(t)$ is the displacement due to the random force. In the following, we neglect the displacement $r_{n}(t)$ for simplicity. Eq. (5) implies that the $n$-th bead is not affected by the force $F_{m}(t)$ until the tension, generated by the force $F_{m}(t)$, diffuses to this bead.

Eq. (5) predicts that in the bulk solution, the force $F_{m}(t)$ that is necessary to displace the $m$-th bead by $u_{m}$ during the loop extrusion process has the form

$$
F_{m}(t)=\frac{\zeta u_{m}}{\sqrt{\pi}} \sqrt{\frac{r \tau_{\mathrm{c}}}{\tau_{1}}} \delta(t)
$$

where $r$ is the duty ratio of cohesin and $\tau_{1}$ is the monomer relaxation time, see eq. (2) with $N=1$. Eq. (6) is an asymptotic form of the extrusion force for $t>r \tau_{\mathrm{c}}$. Eq. (6) is derived by assuming that cohesin generates a constant force on the $m$-th bead only during the time $r \tau_{\mathrm{c}}$ and that the force displaces the bead by a distance $u_{m}$, see the first paragraph of sec. S3 in the Supplementary Material. The bead is freely mobile for $t>r \tau_{c}$, reflecting the fact that the bead is in the bulk solution. At the interface, the force $F_{m}(t)$ has the form

$$
F_{m}(t)=\frac{1}{\pi^{3 / 2}} \frac{1}{\sqrt{\tau_{1}}} \frac{\zeta u_{m}}{\sqrt{t}}
$$

Eq. (7) is the force to displace the $m$-th bead by a distance $u_{m}$ and keep the bead at the position for time $t$, see the first and second paragraphs of sec. S2 in the Supplementary Material for the derivation. The interface is introduced in the boundary condition, with which cohesin and the bead occupied by the cohesin are entrapped at $z=0$ until the cohesin extrudes the next bead.

By using eqs. (5), (6), and $u_{m}=z_{m-1}\left(m \tau_{\mathrm{c}}\right)-z_{m}\left(m \tau_{\mathrm{c}}\right)$, the position $z_{n}(t)$ of the $n$-th bead at time $t\left(m \tau_{\mathrm{c}}<t<\right.$ $\left.(m+1) \tau_{\mathrm{c}}\right)$ is derived in the form

$$
\begin{aligned}
z_{n}(t)-z_{n}(0)= & -\sum_{l=2}^{m}\left(z_{l}\left(l \tau_{\mathrm{c}}\right)-z_{l-1}\left(l \tau_{\mathrm{c}}\right)\right) \\
& \times K\left(n, l, t-l \tau_{\mathrm{c}}\right),
\end{aligned}
$$

with the function $K(n, m, t)=\sqrt{\frac{r \tau_{c}}{\pi \tau_{1}}} G(n, m, t)$ for the bulk solution, see sec. S3 in the Supplementary Material for the derivation. The form of the position $z_{n}(t)$ for the interface is derived by using $z_{l-1}\left(l \tau_{\mathrm{c}}\right)=0$ and $K(n, m, t)=\operatorname{erfc}\left(\frac{\pi(n-m) \sqrt{\tau_{1}}}{2 \sqrt{t}}\right)$ to eq. (8) $(\operatorname{erfc}(x)=$ $\frac{2}{\sqrt{\pi}} \int_{x}^{\infty} d t \mathrm{e}^{-t^{2}}$ is the complementary error function), see sec. S2 in the Supplementary Material for the derivation. By using eq. (8), one derives the relationship

$$
z_{m}\left(m \tau_{\mathrm{c}}\right)-z_{m-1}\left(m \tau_{\mathrm{c}}\right)=\sum_{l=1}^{m} \Gamma_{m l}\left(z_{l}(0)-z_{l-1}(0)\right),
$$

where $\Gamma_{m n}$ is the solution of the equation

$$
\begin{aligned}
\Gamma_{m n}= & \delta_{m n}-\sum_{l=n}^{m-1} \Gamma_{l n}\left[K\left(m, l,(m-l) \tau_{\mathrm{c}}\right)\right. \\
& \left.-K\left(m-1, l,(m-l) \tau_{\mathrm{c}}\right)\right] .
\end{aligned}
$$

The position $z_{n}(t)$ is derived as a function of the positions $z_{l}(0)(l=1,2, \cdots, n)$ of the beads at $t=0$ by substituting eq. (9) into eq. (8).

The mean square distance $\left\langle P^{2}(t)\right\rangle$ between the starting and ending sites

$$
\left\langle P^{2}(t)\right\rangle=\left\langle\left(z_{N}(t)-z_{m=t / \tau_{c}}(t)\right)^{2}\right\rangle
$$

is derived by using the initial condition, with which the chain is ideal for $t=0(\langle\rangle$ is the average with respect to the initial positions of the beads). Indeed, at the interface, the initial distribution of the beads is not Gaussian because the beads of the chain are not distributed equally to the two domains [18]. However, we here use the Gaussian beads distribution for both in the bulk and at the interface to highlight the roles played by the entrapment of the beads to the dynamics of the chain.

Results. - The mean square distance $\left\langle P^{2}(t)\right\rangle$ is a function of the number $N$ of beads in the looping region and the ratio of the time scales that is defined by

$$
\alpha=\frac{\tau_{N}}{\tau_{\mathrm{ex}}},
$$

where $\tau_{\text {ex }}\left(=N \tau_{\mathrm{c}}\right)$ is the time scale of the loop extrusion ${ }_{188}$ process. 


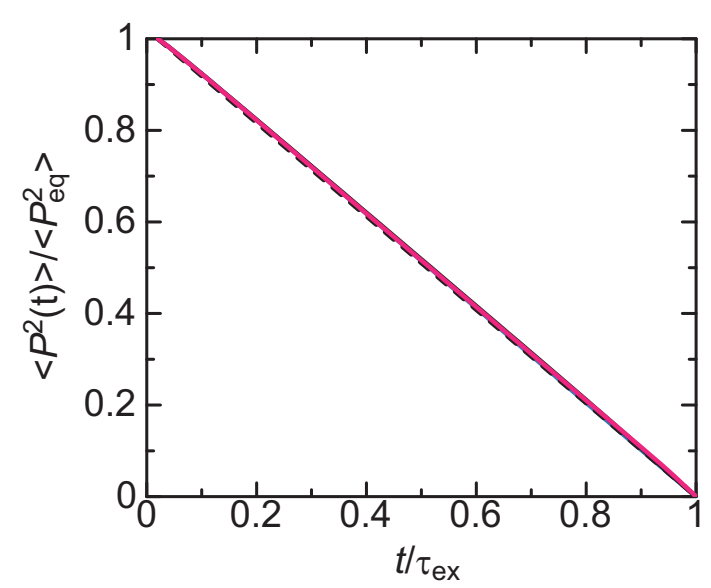

Fig. 3: The mean square distance $\left\langle P^{2}(t)\right\rangle$ between the starting and ending sites of the loop extrusion process (rescaled by the equilibrium value) is shown as a function of time $t$ (rescaled by the time scale $\tau_{\text {ex }}$ of loop extrusion) for $\alpha=0.1$ (cyan), 1.0 (black), and 5.0 (magenta) ( $\alpha$ is defined by eq. (12)) when the chain is in a bulk solution. The broken curves are the local equilibrium values. These curves are derived by numerically calculating eq. (10). We used $N=50$ and $r=0.5$ for the calculations.
Our theory predicts that in the bulk solution, the mean square distance $\left\langle P^{2}(t)\right\rangle$ decreases approximately with a constant rate, as soon as the cohesin starts the loop extrusion process. This is because the loop extrusion process stretches the chain region between the site embraced by cohesin and the ending site, and the elastic force generated by this process displaces the starting site towards the ending site, see fig. 2. In the first approximation, the mean square distance is thus the local equilibrium value,

$$
\frac{3\left\langle P^{2}(t)\right\rangle}{N b^{2}} \simeq 1-\frac{t}{\tau_{\mathrm{ex}}} .
$$

The mean square distance is indeed slightly larger than the local equilibrium value because the chain is stretched by the loop extrusion process, see fig. 3. The deviation $\left\langle\delta P^{2}(t)\right\rangle$ from the local equilibrium value increases with time until the tension generated by the loop extrusion process arrives at the ending site, see fig. 4. The time at which the tension arrives at the ending site decreases with increasing the ratio $\alpha$. It is because the distance by which the tension propagates scales as $t^{1 / 2}$ and the distance by which the cohesin extrudes as $t$. The deviation $\left\langle\delta P^{2}(t)\right\rangle$ has an asymptotic form

$$
\begin{aligned}
\frac{3\left\langle\delta P^{2}(t)\right\rangle}{N b^{2}}= & -\frac{r}{2 \pi \alpha \beta}\left(1-\mathrm{e}^{2 N \beta t / \tau_{\text {ex }}}\right) \\
& \times\left(1-\mathrm{e}^{-\left(\pi^{2} \alpha+N \beta\right)\left(1-t / \tau_{\text {ex }}\right)}\right)^{2}
\end{aligned}
$$

for large values of the ratio $\alpha$ (with $\beta=\log (\sqrt{r} / 2)$ ).

At the interface, the mean square distance $\left\langle P^{2}(t)\right\rangle$ does not change when the cohesin starts the loop extrusion

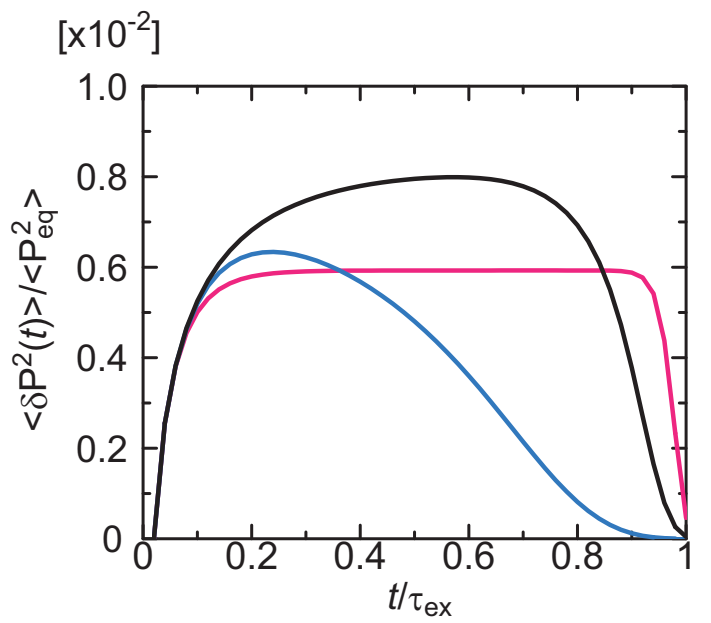

Fig. 4: The deviation $\left\langle\delta P^{2}(t)\right\rangle$ of the mean square distance from the local equilibrium value (the difference between the broken curve and the curves of corresponding color in fig. 3) is shown as a function of time $t$ (rescaled by the time scale $\tau_{\text {ex }}$ of the loop extrusion process) for $\alpha=0.1$ (cyan), 1.0 (black), and 5.0 (magenta), where $\alpha$ is defined by eq. (12). These curves are derived by numerically calculating eq. (10). We used $r=0.5$ and $N=50$ for the calculations.

process, see fig. 5. This is because the starting site is entrapped at the interface and thus the mean square distance $\left\langle P^{2}(t)\right\rangle$ does not change until the tension generated by the loop extrusion arrives at the ending site. The time at which the tension arrives at the ending site decreases with increasing the ratio $\alpha$. For large values of $\alpha$, the mean square distance $\left\langle P^{2}(t)\right\rangle$ has an asymptotic form

$$
\frac{3\left\langle P^{2}(t)\right\rangle}{N b^{2}}=1-\frac{4}{\pi^{2}} \frac{N}{\alpha}\left(2 \frac{t}{\tau_{\text {ex }}}-1\right) \mathrm{e}^{-\pi^{2} \alpha\left(1-t / \tau_{\text {ex }}\right)}
$$

for $t / \tau_{\mathrm{ex}}>1 / 2$ and $\frac{3\left\langle P^{2}(t)\right\rangle}{N b^{2}}=1$ for $t / \tau_{\mathrm{ex}}<1 / 2$. For small values of $\alpha$, the mean square distance asymptotically approaches the local equilibrium value, see eq. (13).

Discussion. - We used an extension of the Rouse model to predict the dynamics of a chain in the bulk solution and at an interface, when the chain is extruded by cohesin with a constant rate. This system has two characteristic features - i) the bead to which forces are applied changes as a function of time and ii) the displacement of the beads due to the loop extrusion process depends on the history of the chain dynamics. The first feature is shared by the problem of polymer translocation through a small pore in a membrane. A scaling theory predicts that the end of the polymer is not influenced by the force applied at the pore until the tension arrives at the end $[19,20]$, analogous to the loop extrusion at the interface. When the ratio $\alpha$ of the time scale is large, the motion of the cohesin is faster than the diffusion of the tension generated at the starting site. The stretching of the chain is amplified as the cohesin extrudes the loop. This second 


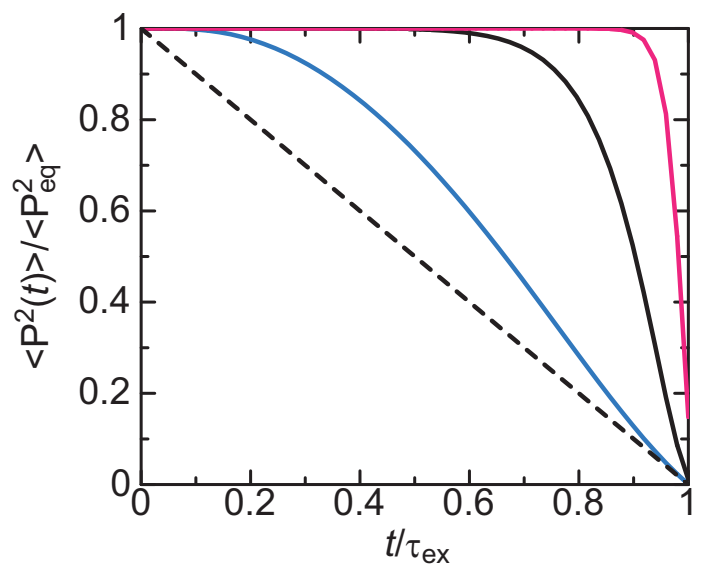

Fig. 5: The mean square distance $\left\langle P^{2}(t)\right\rangle$ between the starting and ending sites (rescaled by the equilibrium value) is shown as a function of time $t$ (rescaled by the time scale of loop extrusion $\left.\tau_{\text {ex }}\right)$ for $\alpha=0.1$ (cyan), 1.0 (black), and 5.0 (magenta). These curves are derived by numerically calculating eq. (10). We used $N=50$ for the calculations.

feature is taken into account in the recursion relationship, eq. (10).

Our theory predicts that the mean square distance between the starting and ending sites of a chain decreases approximately with a constant rate in the bulk. In contrast, at an interface, the mean square distance does not change until the tension generated by the loop extrusion process diffuses to the ending site. The difference of the mean square distance between the chains in the bulk and those at an interface increases with increasing the rate of the loop extrusion process. Recent simulations showed that the size of the extruded loops decreases with increasing the rate of the loop extrusion process [12]. These features reflect the non-equilibrium nature of this process.

In our theory, we have used a couple of assumptions: i) we model the chromatin fiber as a Rouse chain that does not take into account the hydrodynamic interactions and excluded volume interactions between the chain segments, ii) we neglected the tension propagation along the loop, which may be significant for the case of the loop extrusion in the bulk solution, iii) cohesin extrudes the chain with a constant rate, iv) the surface tension is large enough so that the shape of the interface is not perturbed by the loop extrusion process, $\mathrm{v}$ ) cohesin is loaded at $t=0$ onto the chain which is at that point in equilibrium, and vi) the displacement due to the random force is omitted by preaveraging eq. (5). These assumptions are useful to simplify the model to highlight the roles played by the entropic elasticity of the chain and the fact that the chain dynamics significantly depends on the mobility of the bead that is embraced by cohesin. We could even derive the analytical form of the mean square distance for large values of $\alpha$, see eqs. (14) and (15). An extension of our theory may be useful to study more biologically (and experimentally) relevant problems, such as the dynamics of the promoter- enhancer binding and the steady state conformation of chromatin at larger scales.

Because of its simplicity, our theory may be better tested by an in vitro experiments. In contrast to cohesin, condensin was shown to act as a molecular motor that extrudes DNA loops $[14,15]$. The Rouse model is (thought to be) effective to treat the dynamics of a chain in a concentrated solution (in which the excluded volume interactions and the hydrodynamic interactions are screened) on long time and length scales [13]. Our theory is thus best tested by an experiment that measures the end-to-end distance (or the radius of gyration) of DNA in a concentrated solution. Taking into account hydrodynamic interactions in an extension of our theory treats the loop extrusion of DNA in a dilute solution. A scaling theory predicts that hydrodynamic interactions (and excluded volume interactions) only change the scaling exponent of the dynamics of tension propagation [21]. This implies that the dynamics of DNA in the bulk solution is very different from that at the interface even when hydrodynamic interactions (and/or excluded volume interactions) are significant. However, it is of interest to theoretically predict how such long-range interactions change our results.

Recent single molecule experiments indicated that cohesin may not show uni-directional motion [22-24]. One experiment suggests that the cohesin ring is not large enough to accomodate two chromatin fibers and thus cohesin molecules have to form dimers to produce a chromatin loop [22]. We have proposed the osmotic mechanism with which the uni-directional motion of cohesin dimers is driven by the osmotic pressure that is generated by cohesin monomers [25]. Marenduzzo and coworkers proposed a similar mechanism, but only with cohesin dimers [26]. The dynamics of a chain extruded by an osmotic mechanism is different from the dynamics of a chain extruded by a motor mechanism in the following points: First, the extrusion rate is a function of time. It also depends on the loading rate of cohesin monomers (and dimers). The dynamics of the chain also influences the dynamics of cohesin dimers. Second, the time scale of the loop extrusion process scales as $N^{2} / D_{\mathrm{c}}$, where $D_{\mathrm{c}}$ is the diffusion constant of cohesin monomers/dimers. Third, when the motion of the two units of a cohesin dimer is completely random, these units may move in the same direction, relative to the starting site. In this case, the dimer does not decrease the average square end-to-end distance significantly. When each unit of a dimer can move in each side of the starting site, dimers decrease the average square end-to-end vector significantly. However, the maximum number of dimers that are loaded on the chain is limited by the distance between the starting site and the domain boundary due to the excluded volume interactions between cohesin monomers/dimers. The uni-directional motion of cohesin may be driven by RNA polymerase (or other motors) that pushes cohesin during transcription [27]. Our theory is effective for the case in which these motors push cohesin all the way along the domain. When a domain is composed 
of multiple transcription units, the dynamics of a cohesin molecule switches between thermal motion and episodes of uni-directional motion caused by transcription.

$$
* * *
$$

This work was supported by JST, PRESTO Grant Number JPMJPR18KA and JSPS Kakenhi Grant Number $18 \mathrm{~K} 03558$.

\section{REFERENCES}

[1] Dixon J. R., Selvaraj S., Yue F., Kim A., Li Y., Shen Y., Hu M., Liu J. S., and ReN B., Nature, 485 (2012) 376.

[2] Nora E. P., Lajoie B. R., Schulz E. G., Giorgetti L., Okamoto I., Servant N., Piolot T., van Berkum N. L., Meisig J., Sedat J., Gribnau J., Barillot E., Blüthgen N., Dekker J. and Heard E., Nature, 485 (2012) 381.

[3] Rao S.S.P, Huntley M.H., Durand N.C., Stamenova E. K., Bochkov I. D., Robinson J. T., Sanborn A. L., Machol I., Omer A. D., Lander E. S., and Aiden E. L., Cell, 159 (2014) 1685.

[4] Sanborn A.L., RaO S.S.P., Huang S.C., Durand N.C., Huntley M.H., Jewett A.I., Bochkov I.D., ChinnapPan D., Cutkosky A., Li J., Geeting K.P. Gnirke A., Melnikov A., McKenna D., Stamenova E.K., Lander E.S., and Aiden E. L., Proc. Nat. Acad. Sci. USA, 112 (2015) E6456.

[5] Fudenberg G., Imakaev M., Lu C., Goloborodko A., Abdennur N., and Mirny L. A., Cell Rep., 15 (2016) 2038.

[6] RaO S.S.P., Huang S.C., St Hilaire B.G., Engreitz J.M. Perez E. M., Kieffer-Kwon K.R., Sanborn A.L., Johnstone S.E., Bascom G.D., BochKov I.D., Huang X., Shamim M.S., Shin J., Turner D., Ye Z., Omer A.D., Robinson J.T., Schlick T., Bernstein B.E., Casellas R., Lander E.S., and Aiden E.L., Cell, 171 (2017) 305.

[7] Doi M., Chem. Phys., 9 (1975) 455.

[8] Guérin T., Bénichou O., and Voituriez, Nat. Chem., 4 (2012) 568.

[9] Sabari B.R., Dall'Agnese A., Boija A., Klein I.A., Coffey E.L., Shrinivas K., Abraham B.J., Hannett N.M., Zamudio A.V., Manteiga J.C., Li C.H., Guo Y.E., Day D.S., Schuijers J., Vasile E., Malik S., Hnisz D., Lee T.I., Cisse I.I., Goeder R.G., SharP P.A., Chakraborty A.K., and Young R.A., Science, 361 (2018) 379.

[10] Boija A., Klein I.A., Sabari B.R., Dall'Agnese A., Coffery E.L., Zamudio A.V., Li C.H., Shrinivas K., Manteiga J.C., Hannett N.M., Abraham B.J., Afeyan L.K., Guo Y.E., Rimel J.K., Fant C.B., Schuijers J., Lee T.I., TaAtjes D.J., and Young R.A., Cell, 175 (2018) 1842.

[11] Shin Y., Chang Y.C., Lee D.S.W., Berry J., Sanders D.W., Ronceray P., Wingreen N.S., HaAtaja M., and Brangwynne C.P., Cell, 175 (2018) 1481.
[12] Nuebler J., Fudenberg G., Imakaev M., Abdennur N., and Mirny L.A., Proc. Nat. Acad. Sci. USA, 115 (2018) E6697.

[13] Dor M. and Edwards S.F., The Theory of Polymer Dynamics (Oxford Univ. Press, Oxford, UK) 1986.

[14] Terakawa T., Bisht S., Eeftens J.M., Dekker C., Hearing C.H., and Greene E.C., Science, 358 (2017) 672 .

[15] Ganji M., Shaltiel I.A., Bisht S., Kim E., Kalichava A., Hearing C.H., and Dekker C., Science, 360 (2018) 102.

[16] Vian L., Pekowska A., Rao S.S.P., Kieffer-Kwon K.R., Jung S., Baranello L., Huang S.C., Khattabi L.E., Dose M., Pruett N., Sanborn A.L., Canela A., Maman Y., Oksanen A., Resch W., Li X., Lee B., Kovalchuk A.L., Tang Z., Nelson S., Pierro M.D., Cheng R.R., Machol I., St Hilaire, B.G., Durand N.C., Shamim M.S., Stamenova E.K., Onuchic J.N., Ruan Y., Nussenzweig A., Levens D., Aiden E.L., and Casellas R., Cell, 173 (2018) 1165.

[17] Dor M., Soft Matter Physics (Oxford Univ. Press, Oxford, UK) 2013.

[18] Lindner M., Nir G., Medalion G., Dietrich H.R.C, Rabin Y., and Garini Y., Phys. Rev. E, 83 (2011) 011916.

[19] Sakaue T., Phys. Rev. E, 76 (2007) 021803.

[20] Sakaue T., Phys. Rev. E, 81 (2010) 041808.

[21] Saito T. and Sakaue T., Phys. Rev. E, 92 (2015) 012601.

[22] Stigler J., Camdere G.O., Koshland D.E. and Greene E.C., Cell Rep., 15 (2016) 988.

[23] Davidson I.F., Goetz D., Molodtsov M.I., Huis In't Veld P.J., Weissmann F., Litos G., Cisneros D.A., Ocampo-Hafalla M., Ladurner R., Uhlmann F., Vaziri A., and Peters J.M., EMBO J., 35 (2016) 2671.

[24] Kanke M., Tahara E., Huis In'T Veld P.J., and NishiYAma T., EMBO J., 35 (2016) 2686.

[25] Yamamoto T. and Schiessel H., Phys. Rev. E, 96 (2017) 030402(R).

[26] Brackley C.A., Johnson J., Michieletto D., Morozov A.N., Nicodemi, M., Cook P.R., and Marenduzzo D., Phys. Rev. Lett., 119 (2017) 138101.

[27] Busslinger G.A., Stocsits R.R., van der LeliJ P., Axelsson E., Tedeschi A., Galjart N., and Peters J.M., Nature, 544 (2017) 503. 